\title{
In vitro antisense therapeutics for a deep intronic mutation causing Neurofibromatosis type 2
}

\author{
Elisabeth Castellanos ${ }^{1}$, Imma Rosas $^{1}$, Ares Solanes ${ }^{2}$, Isabel Bielsa ${ }^{3}$, Conxi Lázaro ${ }^{4}$, Cristina Carrato ${ }^{5}$, \\ Cristina Hostalot $^{6}$, Pepe Prades ${ }^{7}$, Francesc Roca-Ribas ${ }^{7}$, Ignacio Blanco ${ }^{2}$ and Eduard Serra ${ }^{\star, 1}$ on behalf of the \\ NF2 Multidisciplinary Clinics HUGTiP-ICO-IMPPC
}

\begin{abstract}
Neurofibromatosis type 2 (NF2) is an autosomal-dominant disorder affecting about 1:33000 newborns, mainly characterized by the development of tumors of the nervous system and ocular abnormalities. Around $85 \%$ of germline NF2 mutations are point mutations. Among them, $\sim 25 \%$ affect splicing and are associated with a variable disease severity. In the context of our NF2 Multidisciplinary Clinics, we have identified a patient fulfilling clinical criteria for the disease and exhibiting a severe phenotype. The patient carries a deep intronic mutation (g. 74409T >A, NG_009057.1) that produces the insertion of a cryptic exon of $167 \mathrm{pb}$ in the mature mRNA between exons 13 and 14 , resulting in a truncated merlin protein (p.Pro482Profs*39). A mutation-specific antisense phosphorodiamidate morpholino oligomer was designed and used in vitro to effectively restore normal NF2 splicing in patient-derived primary fibroblasts. In addition, merlin protein levels were greatly recovered after morpholino treatment, decreasing patient's fibroblasts in vitro proliferation capacity and restoring cytoeskeleton organization. To our knowledge, this is the first NF2 case caused by a deep intronic mutation in which an in vitro antisense therapeutic approximation has been tested. These results open the possibility of using this approach in vivo for this type of mutation causing NF2.
\end{abstract}

European Journal of Human Genetics (2013) 21, 769-773; doi:10.1038/ejhg.2012.261; published online 28 November 2012

Keywords: hereditary cancer; Neurofibromatosis type 2; NF2; antisense therapeutics; deep intronic mutation; morpholino

\section{INTRODUCTION}

Neurofibromatosis type 2 (NF2; MIM ID\#101000) is an autosomaldominant cancer syndrome caused by mutations in the NF2 gene, located on chromosome 22q12. NF2 has an incidence of 1 in 33000 live births showing a wide phenotypic variability and a nearly complete penetrance by the age of $60 .^{1}$ The NF2 gene codes for the tumorsuppressor protein merlin $(69 \mathrm{kDa})$. Merlin regulates cellular processes that are found to be altered in tumorigenesis including: cell-cell adhesion, cytoskeletal architecture and membrane protein organization. $^{2,3}$ NF2 patients are predisposed to develop lesions of the nervous system, eyes and skin. The presence of bilateral vestibular nerve schwannomas is the most distinctive feature of NF2, but patients can develop other clinical manifestations such as schwannomas in other cranial, spinal and peripheral nerves, and also other types of tumors, like meningiomas (both intracranial and intraspinal) and ependymomas (low-grade central nervous system malignancies). Affected individuals can also show peripheral neuropathies (independently of compressive tumors), cataracts, epiretinal membranes, retinal hamartomas and cutaneous tumors, usually schwannomas. ${ }^{4}$ Over $50 \%$ of patients are familial cases and the other $50 \%$ bear de novo mutations. A minimum of $25-33 \%$ of these NF2 sporadic cases are mosaic, ${ }^{5,6}$ which complicates clinical diagnostics and genetic testing. ${ }^{7}$
NF2 patients exhibit a wide mutational spectrum with mutation type frequencies that vary slightly depending on the presence or absence of mosaicism. Globally, around $15 \%$ of germline NF2 mutations are large alterations (from single exon to whole gene deletions) and the remaining $85 \%$ are point mutation. ${ }^{1,8,9}$ In general, constitutional nonsense and frameshift NF2 mutations are associated with severe disease manifestations while, missense mutations and large deletions with milder forms of the disease. Furthermore, somatic mosaicism is often associated with mild phenotypes, regardless of the type of the mutation. Around $25 \%$ of point mutations seem to affect the correct splicing of the NF2 gene ${ }^{10}$ and are associated with a variable disease severity. ${ }^{1,11-13}$

Deep intronic mutations are estimated to count $1-5 \%$ of constitutional mutations in human mendelian diseases. ${ }^{14}$ Such mutations have also been found in NF2 (ref. 15) (3 among 292 unrelated NF2 patients, Ludwine Messiaen Personal Communication). The effect of this type of mutations on mRNA is the inclusion of a part of an intron as a consequence of the creation of a novel donor or acceptor site or an exonic splicing enhancer site, which in conjunction with a nearby cryptic splice, defines a cryptic exon that is included by the spliceosome into mature mRNA. Unlike most intragenic mutations, deep intronic mutations do not alter exons and splicing sites of

${ }^{1}$ Hereditary Cancer Program, Institute of Predictive and Personalized Medicine of Cancer (IMPPC), Badalona, Spain; ${ }^{2}$ Hereditary Cancer Program, Genetic Counseling Unit, Institut Català d'Oncologia (ICO), Badalona, Spain; ${ }^{3}$ Department of Dermatology, Hospital Germans Trias i Pujol (HUGTiP), Badalona, Spain; ${ }^{4}$ Hereditary Cancer Program, Molecular Diagnostic Unit, Institut Català d’Oncologia (ICO-IDIBELL), Badalona, Spain; ${ }^{5}$ Department of Pathology, Hospital Germans Trias i Pujol (HUGTiP), Badalona, Spain; ${ }^{6}$ Department of Neurosurgery, Hospital Germans Trias i Pujol (HUGTiP), Badalona, Spain; ${ }^{7}$ Department of Otolaryngology, Hospital Germans Trias i Pujol (HUGTiP), Badalona, Spain

${ }^{*}$ Correspondence: Dr E Serra, Institut de Medicina Predictiva i Personalitzada del Càncer, Ctra. de Can Ruti, Camí de les Escoles s/n, 08916 Badalona, Barcelona, Spain. Tel: +34 9355430 67; Fax: +34 9346514 72; E-mail: eserra@imppc.org

Received 25 July 2012; revised 27 September 2012; accepted 17 October 2012; published online 28 November 2012 
the gene. Thus, there is the possibility to reverse the effect of such mutations by targeting the mutated sequence with specific antisense oligonucleotides (AONs) ${ }^{16,17}$ like phosphorodiamidate morpholino oligomers (PMOs). PMOs are analogs of oligonucleotides with a six-membered morpholino ring replacing the ribose or deoxyribose backbone, and uncharged phosphorodiamidate intersubunit linkages. In addition to high binding specificity, stability and resistance to nucleases, PMOs show long-lived activity. ${ }^{18}$ The masking of a newly created splice site by AONs prevents the splicing machinery from recognizing the cryptic exon and promotes normal splicing. The first indication that AONs could be used as therapeutic agents for genetic diseases was obtained from studies in $\beta$-thalassemia, in which abnormal splicing of the $\beta$-globin gene due to activation of intronic cryptic sites was corrected using an AON approach. ${ }^{19}$ Later this technology was used successfully for other diseases like cystic fibrosis ${ }^{20}$ or more recently for NF1. ${ }^{21}$ Today, different pre-clinical and clinical splice-modulating therapies using AONs, like restoring open reading frames, influencing alternative splicing or inducing exon inclusion, have been successfully used for many different genetic disorders. ${ }^{16,17}$

In this study, we report a NF2 patient with bilateral vestibular schwannoma who was found to bear a deep intronic mutation in the NF2 gene. We reasoned that the effect of this mutation on splicing could be reverted by the use of AONs and decided to test this possibility in vitro by using patient-derived fibroblasts.

\section{MATERIALS AND METHODS}

\section{Patient description}

The patient studied was born from healthy unrelated parents, and suffered hearing loss in the right ear at the age of 14 because of the development of a vestibular schwannoma. Later on, the patient developed a contralateral vestibular schwannoma causing deafness at the age of 42 and 10 years later, a cerebral schwannoma (a schwannoma of the medulla oblongata). In addition, the patient also developed several intramedullary spinal schwannomas. Following the Manchester Clinical Diagnostic Criteria for NF2,22 this individual was included in a molecular genetic testing program. The patient passed away in 2011, at the age of 52 .

\section{Primary cell culture}

Peripheral blood lymphocytes were isolated using Lymphocyte Isolation Solution (Rafer, Zaragoza, Spain) according to the manufacturer's instructions. Short-term lymphocytes cultures were established using RPMI medium (PAA), $10 \%$ fetal bovine serum (FBS Gold; PAA, Pasching, Austria), 0.2\% Phytohemagglutinin (Invitrogen, Paisley, UK) and penicillin/streptomycin (PAA) at $37^{\circ} \mathrm{C}$ and $5 \% \mathrm{CO}_{2}$. Lymphocytes were treated with $250 \mu \mathrm{g} / \mathrm{ml}$ puromycin (Sigma-Aldrich, St Louis, MO, USA) $4-6 \mathrm{~h}$ before RNA extraction to circumvent the nonsense-mediated decay (NMD) mechanism. ${ }^{23}$

For fibroblast isolation, skin was cut into small pieces and digested with $160 \mathrm{U} / \mathrm{ml}$ collagenase type 1 (Worthington, Lakewood, NJ, USA) and $0.8 \mathrm{U} / \mathrm{ml}$ dispase grade 1 (Worthington). ${ }^{24}$ Fibroblast were grown in Dulbecco's modified Eagle's medium (DMEM; PAA), 10\% fetal bovine serum (FBS Gold; PAA) and penicillin/streptomycin (PAA) at $37^{\circ} \mathrm{C}$ and $5 \% \mathrm{CO}_{2}$.

\section{DNA and RNA preparation and RT-PCR}

Total DNA from peripheral blood was extracted using Flexigene DNA KIT (Qiagen, Hilden, Germany) and DNA from patient-derived cultured fibroblasts was extracted using QIAamp DNA Mini KIT (Qiagen), according to the manufacturer's instructions.

RNA was isolated from both short-term lymphocyte cultures and patient-derived primary fibroblasts using Tripure isolation reagent (Roche, Mannheim, Germany). Total RNA $(\sim 3.5 \mu \mathrm{g})$ was reverse-transcribed using SuperScript II reverse transcriptase (Invitrogen) and random hexamers (Invitrogen).

\section{Mutation analysis}

Mutation characterization in this patient was performed at both RNA and DNA levels, enabling us to gain insight into the nature of the DNA mutation and its effect on mRNA. For mutation analysis, the entire coding region of NF2 was amplified from cDNA in two overlapping PCR fragments (primer sequences available on request). PCR products were sequenced. Alterations detected at cDNA level were also characterized at the genomic level by amplifying and direct sequencing the corresponding DNA region.

The strength of the cryptic exon splice sites was analyzed using Splice Site Prediction by Neural Network from the Berkeley Drosophila Genome Project (http://www.fruitfly.org/seq_tools/splice.html).

Human Genome Variation Society (www.hgvs.org) nomenclature guidelines were used to name the mutation at the DNA level, its effect at the mRNA level, and the predicted resulting protein. The first nucleotide of the ATG translation initiation codon is denoted position +1 according to the NF2 mRNA sequence NM_000268.3 and NM_016418.5.

\section{PMO design and treatment conditions}

The 25-mer mutation-specific PMO (MS-PMO) (5'-CATCCCTCAAATCTCTT ACCGTTCT- $\left.3^{\prime}\right)$ was designed, synthesized and purified by Gene Tools (Philomath, OR, USA). It was designed to target the newly created aberrant splice site by the NF2 mutation. Endo-Porter (Gene Tools) was used to deliver PMOs into cells, according to the manufacturer's instructions.

For the PMO treatment of patient-derived fibroblasts, cells were seeded at $3 \times 10^{5}$ cells per well, in a six-well plate. The next day, culture medium was replaced with fresh 10\% FBS/DMEM medium containing the indicated a

NF2 cDNA in NF2 patient NF2

PATIENT CONTROL

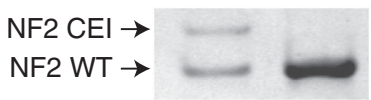

cryptic exon inclusion (CEI)

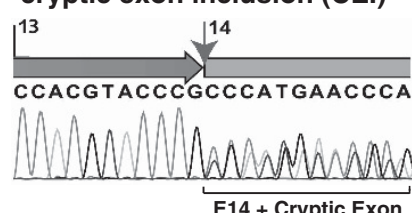

b Deep intronic NF2 mutation scheme c.1447-240 T>A

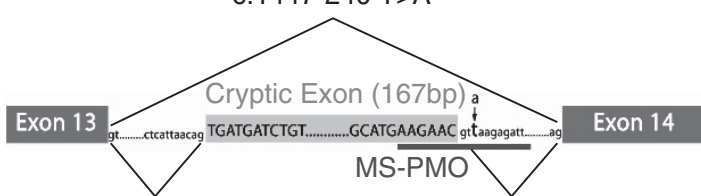

Figure 1 Deep intronic NF2 mutation description. (a) Analysis of patient fibroblasts showed a proportion of NF2 transcripts containing the inclusion of a cryptic exon (NF2 CEI) compared with the WT NF2 mRNA (NF2 WT) (upper panel). Forward sequence of cryptic exon inclusion is shown (bottom panel). (b) Schematic representation of the identified NF2 deep intronic mutation and MS-PMO location. Constitutive and cryptic exons are represented by dark and light gray boxes, respectively. The boundaries of the cryptic inserted exon are shown in uppercase; flanking intronic sequences are shown in lowercase. MS-PMO sequence is underlined. Mutated nucleotide is shown in bold and the nucleotide change is indicated by an arrow. 
concentrations of PMOs (see Results section). Immediately after, Endo-Porter $(6 \mu \mathrm{M})$ was added and mixed.

Control PMO (CTL-PMO) and Endo-Porter alone were used as control treatments. CTL-PMO was designed to target a distinct part of the genome. Each experiment was performed in triplicate.

Merlin western blot and merlin functional analysis

Cell lysates containing $8 \mu \mathrm{g}$ of protein were prepared for western blot analysis of total Merlin. Samples were loaded onto 12\% SDS-PAGE polyacrilamide gels, electrophoresed and transferred to $0.45 \mu \mathrm{m}$ PVDF membrane (Whatman, GE Healthcare, Buckinghamshire, UK). The membrane was blocked with $5 \%$ nonfat dry milk and incubated overnight at $4{ }^{\circ} \mathrm{C}$ with primary antibody, antimerlin A-19 (1/200, SantaCruz Biothechnology, Inc., Santa Cruz, CA, USA). This was followed by incubation with HRP-conjugated secondary antibody at room temperature for $1 \mathrm{~h}$. The blot was developed using the Immun-Star WesternC kit (BIO-RAD, Hercules, CA, USA). Actin was included as a control for equal protein loading. Actin was detected using an anti-actin antibody, ab49900-100 (1/5000) (Abcam, Cambridge, UK).

Cell proliferation was determined by measuring BrdU incorporation for $3 \mathrm{~h}$ in CTL-PMO and MS-PMO treated cells and analyzed by immunofluorescence using mouse anti-BrdU $6 \mu \mathrm{g} / \mathrm{ml}$ (BD Pharmingen, BD Biosciences, Franklin Lakes, NJ, USA).
NF2-muated fibroblasts were stained with Alexa Fluor594 phalloidin (Molecular Probes, Invitrogen, Paisley, UK) following company recommendations to detect and analyze F-actin distribution after CTL-PMO and MS-PMO treatment for $48 \mathrm{~h}$.

\section{RESULTS}

NF2 deep intronic mutation characterization

Sequence analysis of PCR products showed the inclusion of a cryptic exon (CEI) of $167 \mathrm{bp}$ in the mature mRNA between exons 13 and 14 in one of the NF2 alleles (c.1446_1477insNG_009057.1:g.74239_74405), resulting in a putative truncating merlin protein (p.Pro482Profs ${ }^{\star} 39$; Figure 1a). DNA from the corresponding region was subsequently sequenced to determine the precise mutation that caused the inclusion of the cryptic exon and this showed a single nucleotide change located 240 bp upstream of exon 14 (g.74409T >A, NG_009057.1). This mutation produces a new donor site, which is recognized by the splicing machinery and induces the insertion of a cryptic exon (Figure 1b).

\section{AON reversion of the NF2 deep intronic mutation effect}

In order to reverse the effect at mRNA level of this NF2 deep intronic mutation in vitro, we designed a mutation-specific

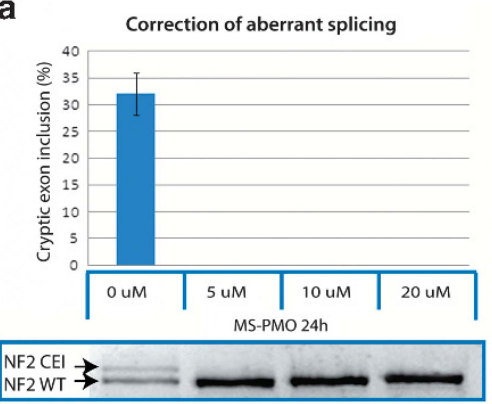

b

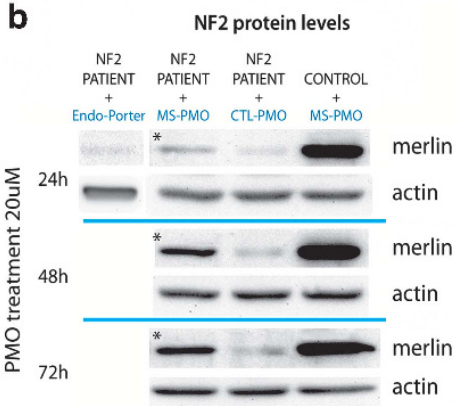

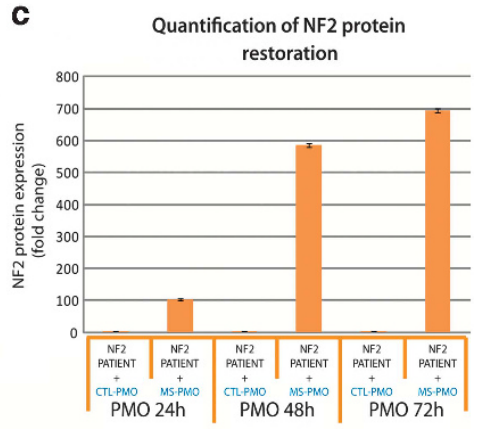
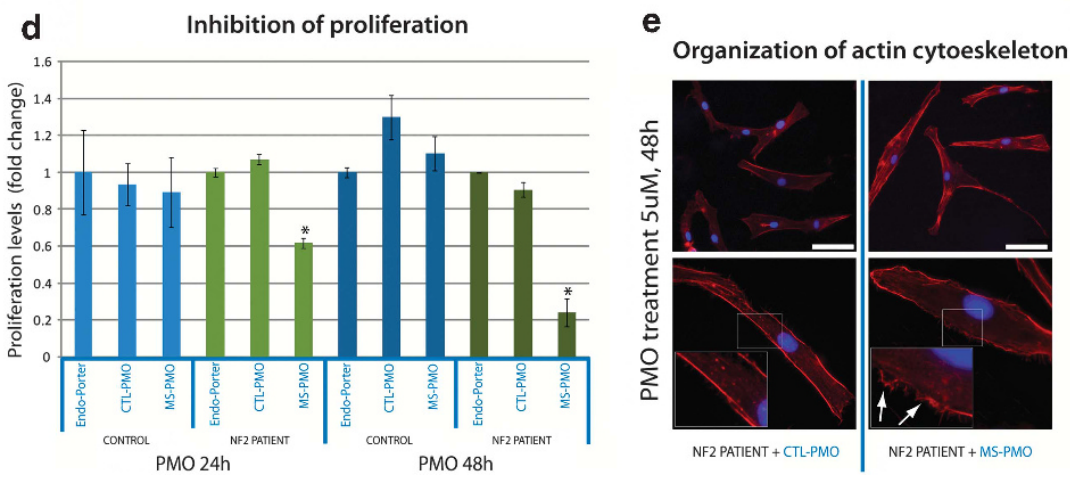

Figure 2 Correcting the effect of the deep intronic NF2 mutation on splicing by PMO treatment. (a) Correction of aberrant splicing by MS-PMO treatment of patient's primary fibroblasts. The treatment was performed using 5, 10 and $20 \mu \mathrm{m}$ PMO concentrations for $24 \mathrm{~h}$. The percentage of the cryptic exoncontaining transcripts (NF2 CEI) in relation to the total (NF2 WT + NF2 CEI) is plotted in the $Y$ axes of the graph. Mean \pm SD is depicted in the bar, representing at least three independent experiments. The corresponding agarose gel electrophoresis is shown below the graph. (b) Representative western blot showing the restoration of merlin protein expression in MS-PMO-treated patient's primary fibroblasts. Patient and control fibroblasts were treated with morpholinos. MS-PMO and CTL-PMO $(20 \mu \mathrm{m})$ were used to treat patient's fibroblast for 24,48 and $72 \mathrm{~h}$. MS-PMO was used to treat control fibroblast using the same conditions. For comparison purposes, a control sample consisting in patient fibroblasts treated with PMO delivery vehicle alone was added for the $24 \mathrm{~h}$ time point. Asterisks highlight merlin recovery. (c) Quantification of merlin expression in NF2-mutant fibroblast cultures after either MS-PMO or CTLPMO $(5 \mu \mathrm{m})$ treatment for 24,48 and $72 \mathrm{~h}$. In the $\mathrm{Y}$ axis, it is plotted the proportion of merlin expression after MS-PMO treatment in relation to merlin expression after CTL-PMO treatment. All merlin values were previously normalized with actin levels. Mean \pm SD is depicted in the bar, representing at least three independent experiments. $\left(^{*}\right)$ Denotes statistical significance using the Mann-Whitney U-test $(P>0.0001)$. (d) Merlin expression level restoration decrease the proliferation capacity of patient's fibroblasts, whereas not interfering in the proliferation capacity of control fibroblasts. Mean \pm SD is depicted in the bar, representing at least three independent experiments. $\left.{ }^{*}\right)$ Denotes statistical significance using the T-test $(P>0.05)$. (e) Cytoskeletal abnormalities of patient's derived fibroblasts were restored after MS-PMO treatment. NF2-mutated fibroblasts treated with CTL-PMO (left panels) showed poorly organized cytoskeleton when compared with the same fibroblast cultures treated with MS-PMO (right panels) at $5 \mu \mathrm{m} \mathrm{PMO} \mathrm{concentration} \mathrm{for} 48 \mathrm{~h}$. Scale bar: $75 \mu \mathrm{m}$. Lower panels display a magnification of a detail of the cellular membrane. Microvilli structures are indicated by arrows. 
phosphorodiamidate morpholino oligomer (MS-PMO) (Figure 1b). A primary skin-derived fibroblast cell culture from the NF2 patient was established to test the ability of MS-PMO to restore normal splicing for this mutation. We observed that without MS-PMO treatment the percentage of aberrantly-spliced transcript (containing the CEI) was approximately $30 \%$ of the total NF2 mRNA (wildtype + aberrantly-spliced transcript). This percentage was not affected by puromycin treatment (data not shown), indicating that NMD mechanism was not influencing the relative percentage of mutated transcripts and probably indicating that the splicing machinery did not always recognize the newly created splicing sites within the intron generating wild-type transcripts from the mutated allele. To set up PMO treatment conditions, first a dose-response experiment was conducted to determine the amount of MS-PMO required for a total correction of mutation effect. Patient-derived fibroblasts were treated with three different PMO concentrations $(5,10$ and $20 \mu \mathrm{M})$ for $24 \mathrm{~h}$, and a complete inhibition of cryptic exon inclusion was already observed at the lowest concentration tested (Figure 2a). The specificity of the MS-PMO was assessed by testing in parallel a Control PMO (CTL-PMO) recognizing a different part of the genome. Only MS-PMO had a corrective effect on aberrant splicing, indicating that its effect was sequence-specific (data not shown). To evaluate the duration of the MS-PMO effect on mutation correction, a time course was performed testing 5 and $20 \mu \mathrm{M}$ PMO concentrations. Transcriptional analysis and merlin quantification was performed at 24, 48 and $72 \mathrm{~h}$ after MS-PMO treatment (Figure 2b). Basal levels of merlin in patient-derived fibroblasts were quite low, despite the fact that only one of the two NF2 alleles was mutated. The levels of merlin in non-transfected patient fibroblasts were very similar to those of PMO delivery vehicle (Endo Porter) treated conditions (data not shown and Figure 2b) A complete decrease in the aberrant transcript was observed at $24 \mathrm{~h}$ at both concentrations (data not shown), but protein levels, although starting to recover at $24 \mathrm{~h}$, were not significantly increased until $48 \mathrm{~h}$ after MS-PMO treatment (Figures $2 \mathrm{~b}$ and $\mathrm{c}$ ). Merlin levels were not affected by CTL-PMO treatment in patient-derived fibroblasts or in fibroblast derived from a non-NF2 individual treated with MS-PMO, further supporting the sequence-specific effect of MS-PMO treatment.

\section{Functional characterization of correct NF2 splicing restoration} Finally, it was important to confirm that correction of aberrant splicing by MS-PMO treatment was also relevant at a functional level. It has been reported that merlin have a role in both actin remodeling and contact-dependent inhibition of proliferation, ${ }^{2,3,25}$ so we decided to measure the effects of MS-PMO treatment on the in vitro fibroblast proliferation capacity and its cytoskeletal reorganization. To determine whether newly restored merlin protein induced by MSPMO could influence the proliferation capacity of patient-derived fibroblasts we performed a BrdU incorporation assay (Figure 2d). Cell proliferation was reduced by $41 \%$ at $24 \mathrm{~h}$ and $67 \%$ at $48 \mathrm{~h}$ comparing MS-PMO and CTL-PMO treatments. No changes were observed in control fibroblasts under the same conditions (data not shown). Next, we examined merlin function through its effect on filamentous actin (F-actin). NF2 patient fibroblasts showed a poorly organized cytoskeleton, both before and after being treated with CTL-PMO (Figure 2e). In contrast, polymerization abnormalities regarding intracellular F-actin were eliminated after MS-PMO treatment with fibroblasts exhibiting well-organized actin stress fibers and abundant apical microvilli (Figure 2e). These results indicated that not only did MS-PMO treatment correct the aberrant splicing but that the correction led to the restoration of merlin levels and activity.

\section{DISCUSSION}

We report here the first NF2 case caused by a deep intronic mutation in which an in vitro antisense therapeutic approximation has been tested. This mutation was detected thanks to the double analysis at the RNA and DNA levels that is performed in our NF2 Multidisciplinary Clinics as a routine genetic testing procedure. The reported mutation would probably be missed if analysis at RNA level were not considered. When a DNA-based strategy is applied in second-generation affected individuals from NF2 families there is a $7 \%$ of mutations that are not detected. ${ }^{1}$ However, it is not known the proportion of this unidentified mutations that lie in deep intronic regions (presumably about 1-2\%, Ludwine Messiaen personal communication).

We show the suitability and sequence-specific action of using AON strategies to correct aberrant splicing caused by this specific deep intronic mutation in the NF2 gene. In addition, this correction also restored merlin levels and wild-type function in at least some cell physiology processes, such as proliferation and cytoeskeleton organization in patient-derived fibroblasts. However, although restoration of merlin levels in Schwann cells (SCs) and Schwannoma-derived SCs have been shown to also inhibit their proliferation capacity and a reorganization of actin cytoskeleton, ${ }^{25-28}$ it should be experimentally proved that an antisense strategy using PMOs is also effective in this cell type, one of the mainly affected in NF2.

This in vitro study using AON technology for NF2 mutations only represent a proof of concept. For a hypothetical use in the clinics, a long path through different phases of preclinical and clinical trials would be required. Recent developments using cell-penetrating peptides conjugated to PMO (PPMO) or the commercially available Vivo-Morpholinos have successfully addressed issues like ensuring efficient AON delivery to affected tissues by improving cellular uptake and avoiding a rapid clearance from the circulation. ${ }^{29,30}$ The success of clinical trials using AON technology for other mis-splicing mutations causing Duchenne muscular dystrophy (DMD) and the efficient delivery of AONs in the nervous system of mouse models for Huntington, Alzheimer and spinal muscular atrophy diseases ${ }^{31-34}$ are encouraging results and indicate a possible path from bench to bedside for this particular type of mutations causing NF2.

Finally, taking into consideration that constitutional nonsense and frameshift NF2 mutations are associated with a severe form of the disease, and that the NF2 gene is composed by many in-frame exons (10 out of 17 exons), the possibility exists of reducing the severity of these mutations for certain exons by forcing their skipping using antisense technology (as it happens for DMD). However, functional studies need to be performed in order to characterize the preserved function of merlin after forcing the partial or total skipping of each in-frame exon (from both alleles) before knowing how many patients could benefit from this hypothetical approach.

\section{CONFLICT OF INTEREST}

The authors declare no conflict of interest.

\section{ACKNOWLEDGEMENTS}

We thank the NF2 patient reported here and his relatives who participated in this study. We thank Juana Fernández-Rodríguez for advice and reagents, Josep Biayna and Bernat Gel for support, Eugeni Aragall and Pilar Armengol for their help with sequencing and also the HUGTiP-IGTP Biobank. This work has been supported by the Institut de Medicina Predictiva i Personalitzada del Cancer (IMPPC); the Government of Catalonia (2009SGR290); and the Spanish Ministry of Science and Innovation, Carlos III Health Institute (ISCIII-RTICC RD06/0020/1051); (PI11/1609). CL, IB and ES thank the 
continuous support from the Spanish association of Patients with Neurofibromatosis (Asociación de Afectados de Neurofibromatosis) and the Asociación Española Contra el Cáncer (AECC).

1 Evans D: Neurofibromatosis type 2 (NF2): a clinical and molecular review. Orphanet $J$ Rare Dis 2009; 19: 16.

2 McClatchey Al, Giovannini M: Membrane organization and tumorigenesis-the NF2 tumor suppressor, Merlin. Genes Dev 2005; 19: 2265-2277.

3 Trofatter JA, MacCollin MM, Rutter JL et al: A novel moesin-, ezrin-, radixin-like gene is a candidate for the neurofibromatosis 2 tumor suppressor. Cell 1993; 72 : 791-800.

4 Asthagiri AR, Parry DM, Butman JA et al: Neurofibromatosis type 2. Lancet 2009; 373: $1974-1986$.

5 Evans DGR, Wallace AJ, Wu CL, Trueman L, Ramsden RT, Strachan T: Somatic mosaicism: a common cause of classic disease in tumor-prone syndromes? Lessons from type 2 Neurofibromatosis. Am J Human Genet 1998; 63: 727-736.

6 Kluwe L, Mautner V, Heinrich B et al: Molecular study of frequency of mosaicism in neurofibromatosis 2 patients with bilateral vestibular schwannomas. J Med Genet 2003; 40: 109-114.

7 Evans DG, Raymond FL, Barwell JG, Halliday D: Genetic testing and screening of individuals at risk of NF2. Clin Genet 2011; 82: 416-424.

8 Ahronowitz I, Xin W, Kiely R, Sims K, MacCollin M, Nunes FP: Mutational spectrum of the NF2 gene: a meta-analysis of 12 years of research and diagnostic laboratory findings. Hum Mutat 2007; 28: 1-12.

9 Baser ME: The distribution of constitutional and somatic mutations in the neurofibromatosis 2 gene. Hum Mutat 2006; 27: 297-306.

10 Ellis Jr. JR, Heinrich B, Mautner VF, Kluwe L: Effects of splicing mutations on NF2transcripts: transcript analysis and information theoretic predictions. Genes Chromosomes Cancer 2011; 50: 571-584.

11 Kluwe L, MacCollin M, Tatagiba M et al: Phenotypic variability associated with 14 splice-site mutations in the NF2 gene. Am J Med Genet 1998; 77: 228-233.

12 Merel P, Hoang-Xuan K, Sanson M et al: Screening for germ-line mutations in the NF2 gene. Genes Chromosomes Cancer 1995; 12: 117-127.

13 Selvanathan SK, Shenton A, Ferner R et al: Further genotype-phenotype correlations in neurofibromatosis 2. Clin Genet 2010; 77: 163-170.

14 Cooper DN, Chen J-M, Ball EV et al: Genes, mutations, and human inherited disease at the dawn of the age of personalized genomics. Hum Mutat 2010; 31: 631-655.

15 De Klein A, Riegman PHJ, Bijlsma EK et al: A G $\rightarrow$ A transition creates a branch point sequence and activation of a cryptic exon, resulting in the hereditary disorder Neurofibromatosis 2. Hum Mol Genet 1998; 7: 393-398.

16 Aartsma-Rus A, den Dunnen JT, van Ommen GJ: New insights in gene-derived therapy: the example of Duchenne muscular dystrophy. Ann N Y Acad Sci 2010; 1214: 199-212.
17 Spitali P, Aartsma-Rus A: Splice modulating therapies for human disease. Cell 2012; 148: $1085-1088$.

18 Summerton J, Weller D: Morpholino antisense oligomers: design, preparation, and properties. Antisense Nucleic Acid Drug Dev 1997; 7: 187-195.

19 Lacerra G, Sierakowska H, Carestia C et al: Restoration of hemoglobin A synthesis in erythroid cells from peripheral blood of thalassemic patients. Proc Natl Acad Sci 2000; 97: 9591-9596.

20 Friedman KJ, Kole J, Cohn JA, Knowles MR, Silverman LM, Kole R: Correction of aberrant splicing of the cystic fibrosis transmembrane conductance regulator (CFTR) gene by antisense oligonucleotides. J Biol Chem 1999; 274: 36193-36199.

21 Pros E, Fernández-Rodríguez J, Canet B et al: Antisense therapeutics for neurofibromatosis type 1 caused by deep intronic mutations. Hum Mutat 2009; 30: 454-462.

22 Evans D, Baser M, O'Reilly B et al: Management of the patient and family with neurofibromatosis 2: a consensus conference statement. Br J Neurosurg 2005; 19: 5-12.

23 Messiaen LM, Callens T, Mortier G et al: Exhaustive mutation analysis of the NF1 gene allows identification of $95 \%$ of mutations and reveals a high frequency of unusual splicing defects. Hum Mutat 2000; 15: 541-555.

24 Dominski Z, Kole R: Restoration of correct splicing in thalassemic pre-mRNA by antisense oligonucleotides. Proc Natl Acad Sci USA 1993; 90: 8673-8677.

25 Lallemand D, Curto M, Saotome I, Giovannini M, McClatchey Al: NF2 deficiency promotes tumorigenesis and metastasis by destabilizing adherens junctions. Genes Dev 2003; 17: 1090-1100.

26 Bashour AM, Meng JJ, Ip W, MacCollin M, Ratner N: The neurofibromatosis type 2 gene product, merlin, reverses the $\mathrm{F}$-actin cytoskeletal defects in primary human Schwannoma cells. Mol Cell Biol 2002; 22: 1150-1157.

27 Morrison H, Sperka T, Manent J, Giovannini M, Ponta H, Herrlich P: Merlin/ Neurofibromatosis type 2 suppresses growth by inhibiting the activation of Ras and Rac. Cancer Res 2007; 67: 520-527.

28 Pelton PD, Sherman LS, Rizvi TA et al: Ruffling membrane, stress fiber, cell spreading and proliferation abnormalities in human Schwannoma cells. Oncogene 1998; 17: 2195-2209.

29 Moulton JD, Jiang S: Gene knockdowns in adult animals: PPMOs and vivo-morpholinos. Molecules 2009; 14: 1304-1323.

30 Muntoni F, Wood MJ: Targeting RNA to treat neuromuscular disease. Nat Rev Drug Discov 2011; 10: 621-637.

31 Erickson MA, Niehoff ML, Farr SA et al: Peripheral administration of antisense oligonucleotides targeting the amyloid-beta protein precursor reverses AbetaPP and LRP-1 overexpression in the aged SAMP8 mouse brain. J Alzheimers Dis 2012; 28: 951-960.

32 Hua Y, Sahashi K, Rigo F et al: Peripheral SMN restoration is essential for long-term rescue of a severe spinal muscular atrophy mouse model. Nature 2011; 478: 123-126.

33 Kordasiewicz HB, Stanek LM, Wancewicz EV et al: Sustained therapeutic reversal of Huntington's disease by transient repression of huntingtin synthesis. Neuron 2012; 74: 1031-1044.

34 Passini MA, Bu J, Richards AM et al: Antisense oligonucleotides delivered to the mouse CNS ameliorate symptoms of severe spinal muscular atrophy. Sci Transl Med 2011; 3: 72ra18. 